\title{
A NOTE ON DUNFORD-PETTIS OPERATORS
}

\author{
by J. R. HOLUB
}

(Received 23 June, 1986)

Talagrand has shown $[4$, p. 76$]$ that there exists a continuous linear operator from $L^{1}[0,1]$ to $c_{0}$ which is not a Dunford-Pettis operator. In contrast to this result, Gretsky and Ostroy [2] have recently proved that every positive operator from $L^{1}[0,1]$ to $c_{0}$ is a Dunford-Pettis operator, hence that every regular operator between these spaces (i.e. a difference of positive operators) is Dunford-Pettis. In this note we prove that the converse is also true, thereby characterizing the Dunford-Pettis operators from $L^{1}[0,1]$ to $c_{0}$ as follows:

Theorem. An operator $T: L^{1}[0,1] \rightarrow c_{0}$ is a Dunford-Pettis operator if and only if $T=T_{1}-T_{2}$ where $T_{1}$ and $T_{2}$ are positive operators.

In order to prove this result we need the following simple representation for bounded linear operators from $L^{1}[0,1]$ to $c_{0}$. As usual, $\left\{e_{n}\right\}_{n=1}^{\infty}$ denotes the basis for $c_{0}$ and $l^{p}$, $p \geqslant 1$, defined by $e_{n}=\left\{\delta_{n i}\right\}_{i=1}^{\infty}$.

Lemma. A linear operator $W: L^{1}[0,1] \rightarrow c_{0}$ is continuous if and only if there is a sequence $\left\{H_{n}\right\}_{n=1}^{\infty}$ in $L^{\infty}[0,1]$ which converges to zero in $w^{*}$-topology and for which $W(f)=\sum_{n=1}^{\infty}\left\langle H_{n}, f\right\rangle e_{n}$ for all $f$ in $L^{1}[0,1]$.

Proof. $(\Rightarrow)$ : If $W$ is continuous from $L^{1}[0,1]$ to $c_{0}$ then for every $f$ in $L^{1}[0,1]$ we have $W(f)=\sum_{n=1}^{\infty}\left\langle W f, e_{n}\right\rangle e_{n}=\sum_{n=1}^{\infty}\left\langle W^{*} e_{n}, f\right\rangle e_{n}$, where $\left\{W^{*} e_{n}\right\}_{n=1}^{\infty}=\left\{H_{n}\right\}_{n=1}^{\infty}$ is a sequence in $L^{\infty}[0,1]$ which is $\mathrm{w}^{*}$-convergent to zero since $W^{*}$ is $\mathrm{w}^{*}$-continuous from $l^{1}$ to $L^{\infty}[0,1]$.

$(\Leftarrow)$ : If such a sequence $\left\{H_{n}\right\}_{n=1}^{\infty}$ exists then $\left\{\left\langle H_{n}, f\right\rangle\right\}_{n=1}^{\infty} \in c_{0}$ for all $f$ in $L^{1}[0,1]$, implying that $\sum_{n=1}^{\infty}\left\langle H_{n}, f\right\rangle e_{n}$ converges in $c_{0}$ for all $f$ in $L^{1}[0,1]$. Hence by the Uniform Boundedness Principle the operator $W$ defined by $W(f)=\sum_{n=1}^{\infty}\left\langle H_{n}, f\right\rangle e_{n}$ is continuous.

Proof of Theorem. $(\Leftarrow)$ : If $T$ is the difference of positive operators then $T$ is a Dunford-Pettis operator by the theorem of Gretsky and Ostroy [2].

$(\Rightarrow)$ : Conversely, suppose $T: L^{1}[0,1] \rightarrow c_{0}$ is a Dunford-Pettis operator. Let $\left\{H_{n}\right\}_{n=1}^{\infty}$ be the corresponding sequence in $L^{\infty}[0,1]$ (equal to $\left\{T^{*} e_{n}\right\}_{n=1}^{\infty}$ ) for which $T(f)=\sum_{n=1}^{\infty}\left\langle H_{n}, f\right\rangle e_{n}$ for all $f$ in $L^{1}[0,1]$, which exists by the preceding Lemma. Since $T$ is Dunford-Pettis it is well-known that if $i: L^{\infty}[0,1] \rightarrow L^{1}[0,1]$ denotes the canonical injection map then the operator $T \circ i: L^{\infty}[0,1] \rightarrow L^{1}[0,1] \rightarrow c_{0}$ is compact [1]. Hence

Glasgow Math. J. 29 (1987) 271-273. 
applying the Banach-Steinhaus theorem [3, p. 86] to the partial sum operators associated with the basis $\left\{e_{n}\right\}_{n=1}^{\infty}$ for $c_{0}$ gives

$$
\lim _{N \rightarrow \infty} \sup _{\substack{\|g\| \leq 1 \\ g \in L^{\alpha}[0,1]}}\left\|\sum_{n=N}^{\infty}\left\langle T \circ i(g), e_{n}\right\rangle e_{n}\right\|=0,
$$

or

$$
\lim _{N \rightarrow \infty} \sup _{\substack{\|f\|<1 \\ g \in L^{\alpha}[0,1]}}\left\|\sum_{n=N}^{\infty}\left\langle i\left(T^{*} e_{n}\right), g\right\rangle e_{n}\right\|=0 .
$$

Since $T^{*} e_{n}=H_{n}$ it follows from the definition of the norm in $c_{0}$ that $\lim _{n \rightarrow \infty} \sup _{\|g\| \leq 1}$ $\left|\left\langle i\left(H_{n}\right), g\right\rangle\right|=0$, and hence that $\lim _{n \rightarrow \infty}\left\|i\left(H_{n}\right)\right\|_{1}=0$, where $\|\cdot\|_{1}$ denotes the norm in $L^{1}[0,1]$.

Now for each $n=1,2, \ldots, H_{n}=H_{n}^{+}-H_{n}^{-}$where each of these is non-negative a.e. on $[0,1]$. Moreover since $\left\|i\left(H_{n}\right)\right\|_{1} \rightarrow 0$ it follows that $\left\|i\left(H_{n}^{+}\right)\right\|_{1} \rightarrow 0$ and $\left\|i\left(H_{n}^{-}\right)\right\|_{1} \rightarrow 0$ as well. But then since $\left\{H_{n}^{+}\right\}$and $\left\{H_{n}^{-}\right\}$are bounded sequences of non-negative functions in $L^{\infty}[0,1]$ it is clear that for any $f$ in $L^{1}[0,1]$ we have $\lim _{n \rightarrow \infty} \int_{0}^{1} H_{n}^{+}(t) f(t) d t=$ $\lim _{n \rightarrow \infty} \int_{0}^{1} H_{n}^{-}(t) f(t) d t=0$.

That is, $\left\{H_{n}^{+}\right\}_{n=1}^{\infty}$ and $\left\{H_{n}^{-}\right\}_{n=1}^{\infty}$ both converge to zero in the $\mathrm{w}^{*}$-topology on $L^{\infty}[0,1]$, implying, by the Lemma, that the operators $T_{1}$ and $T_{2}$ from $L^{1}[0,1]$ to $c_{0}$ defined by $T_{1}(f)=\sum_{n=1}^{\infty}\left\langle H_{n}^{+}, f\right\rangle e_{n}$ and $T_{2}(f)=\sum_{n=1}^{\infty}\left\langle H_{n}^{-}, f\right\rangle e_{n}$ are both continuous. Since $T_{1}$ and $T_{2}$ are clearly positive and $T=T_{1}-T_{2}$, the proof is complete.

REMARKs. It turns out that the result of Gretsky and Ostroy actually holds for a positive operator $T: L^{1}(\mu) \rightarrow c_{0}$, where $\mu$ is any $\sigma$-finite measure. The proof in this general case is, of necessity, very different from that given by Gretsky and Ostroy for the case $L^{1}[0,1]$ owing to the fact that the characterization of Dunford-Pettis operators given in [1] and used in their proof (and in that of the Theorem above) does not apply in the more general case. This result and others related to the ideas in this note (e.g., if $X$ is any separable Banach space in which weak and norm convergence of sequences are not the same then there is a non-Dunford-Pettis operator $T: X \rightarrow c_{0}$ ) will be given in a later paper devoted to a more comprehensive study of Dunford-Pettis operators then we have attempted here.

\section{REFERENCES}

1. J. Bourgain, Dunford-Pettis operators on $L^{1}$ and the Radon-Nikodym property, Israel $J$. Math. 37 (1980), 27-34. 
2. N. Gretsky and J. Ostroy, The compact range property and $c_{0}$, Glasgow Math. J. 28 (1986), $113-114$.

3. H. Schaefer, Topological vector spaces (Macmillan, 1966).

4. M. Talagrand, Pettis integral and measure theory, Mem. Amer. Math. Soc. 307 (1984).

Virginia Polytechnic Institute

AND STATE UNIVERSITY

Blacksburg Virginia 24061 